\title{
Prioridades de investigación en la Obra social de empleados públicos, Mendoza, Argentina. Un estudio Delphi sobre producción de conocimientos en la seguridad social
}

\section{Cecilia Amalia Molina}

Doctora en Ciencias Sociales. Licenciada en Trabajo Social

Obra Social de los Empleados Públicos. Mendoza, Argentina

https://orcid.org/0000-0001-5569-786X • cecilia.molina@osep.mendoza.gov.ar

\section{Resumen}

La investigación en servicios de salud contribuye a informar de manera fundada los desafíos asistenciales, financieros y de gestión que afrontan las organizaciones de la seguridad social. Acordar qué y para qué investigar propicia que decisores políticos y equipos de trabajo se involucren en la producción de conocimientos científicos sobre problemas concretos que deben ser resueltos para mejorar las capacidades de respuesta de los sistemas de salud. Los objetivos de este escrito son compartir los resultados de la aplicación de una técnica de búsqueda de consensos sobre criterios y temas a investigar en la "Obra Social de Empleados Públicos de Mendoza, Argentina".

La metodología empleada es un estudio Delphi del que participaron referentes organizacionales con diferentes formaciones disciplinares y desempeños en los distintos niveles de gestión. Los tópicos priorizados son los modelos de atención de la salud innovadores, centrados en las personas; la identificación de necesidades de salud de los asegurados que no acceden los servicios y la eliminación de barreras de acceso a partir de nuevas tecnologías, como la teleasistencia.

Se concluye que la metodología empleada posibilita que equipos de salud que no trabajan de manera articulada puedan reconsiderar intereses particulares a la luz de intereses institucionales y aportar a los decisores insumos para su tarea.

Palabras clave: Prioridades en salud; Seguridad social; Investigación cualitativa; Método Delphi.

Recibido: 09/01/2021 |Aprobado: 05/05/2021 | Publicado: 01/07/2021

(1)(2) Esta obra está bajo una Licencia Creative Commons Atribución-NoComercialCompartirIgual 4.0 Internacional.

Financiación o proveniencia del artículo: Artículo derivado del estudio Prioridades de investigación en la obra social de empleados públicos, Mendoza, Argentina. Un estudio Delphi sobre producción de conocimientos en la seguridad social, avalado por el Comité de Investigación y Docencia de la Obra Social de Empleados públicos de la provincia de Mendoza, Argentina. Noviembre de 2019 - Febrero de 2020.

¿Cómo citar este artículo? / How to quote this article?

Molina, C. A. (2021). Prioridades de investigación en la obra social de empleados públicos, Mendoza, Argentina. Un estudio Delphi sobre producción de conocimientos en la seguridad social. Prospectiva. Revista de Trabajo Social e intervención social, (32), 223-238. doi: 10.25100/ prts.v0i32.10917. 


\title{
Research Priorities in the Social Work of Public Employees of Mendoza, Argentina. A Delphi Study in Knowledge Production in Social Security
}

\begin{abstract}
Research in health services contributes information and evidence about the challenges of service, finances, and administration that social security organizations face. Agreeing on what to research and for what purpose guides policymakers and work teams in the production of scientific knowledge about specific problems that must be solved to improve the healthcare system's capacity to respond. The aim of this work is to share the results of applying a technique of reaching consensus on the criteria and topics to investigate within the "Obra Social de Empleados Públicos de Mendoza, Argentina".

The applied methodology is a Delphi study with the participation of organizational referents with different disciplinary backgrounds and performances within the different administrative levels. The prioritized topics are innovative healthcare models focused on people; the identification of the needs of those insured who do not access services; and the removal of barriers related to access, with the help of new technologies, such as telecare.

It is concluded that this methodology enables those work teams that are not working articulately to reconsider particular interests in the light of institutional interests and provide well-founded information to policymakers.
\end{abstract}

Keywords: Healthcare priorities; Social security; Qualitative study; Delphi method.

Sumario: 1. Introducción, 2. Metodología, 3. Hallazgos, 4. Conclusiones, 5. Referencias bibliográficas. 


\section{Introducción}

El sector de las obras sociales en Argentina confluye en un sistema de salud que tiene como rasgos centrales la fragmentación del financiamiento, la segmentación en la prestación de servicios y que, como consecuencia, genera profundas desigualdades en el acceso de los ciudadanos a la atención médica. A diferencia de sistemas de otros países latinoamericanos como Costa Rica o Uruguay que en las últimas décadas implementaron reformas tendientes a integrar funciones para avanzar en la equidad, "la seguridad social, en el país, mantiene un patrón divergente en el acceso y la cobertura de salud" (Cetrángolo y Goldschmit, 2018, p. 3) $)^{1}$.

Integran el sistema de obras sociales argentino las obras sociales nacionales, sindicales, de regímenes especiales como las del personal de las Fuerzas Armadas, de las universidades y el Instituto Nacional de Seguridad Social para jubilados y pensionados asi como las obras sociales provinciales (OSP). Son cerca de 300 aseguradoras que tienen a cargo cerca del $60 \%$ de la población del país con diferentes recursos, de acuerdo al ingreso promedio de sus contribuyentes. Y "si bien existe un piso prestacional llamado Programa Médico Obligatorio, no siempre se cumple o las prestaciones no se brindan en tiempo y forma" (De Ponti, Canale y Monteferrario, 2016, p. 2). La organización político-administrativa argentina complejiza el problema de la equidad en salud. Indicadores sanitarios básicos como la mortalidad infantil reflejan a las claras desigualdades según dónde reside la población. De ello resulta que mientras menor es el desarrollo económico de las provincias y los municipios, las demandas sanitarias insatisfechas son mayores.

En este escenario heterogéneo, 24 obras sociales provinciales -entre ellas la Obra Social de Empleados Públicos de la provincia de Mendoza (OSEP)-, deben garantizar el acceso igualitario a la salud de los trabajadores estatales y sus familias, quienes representan el 16 $\%$ de la población del país. Estas organizaciones se orientan por el principio de solidaridad, según el cual cada asegurado aporta con relación a su ingreso y recibe según las prestaciones que necesita ${ }^{2}$, pero no asignan los mismos recursos a la salud de los asegurados a su cargo

1 En Argentina, las obras sociales se ocupan de la atención de la salud-enfermedad de la población trabajadora y de los jubilados. De manera más amplia, la Seguridad Social comprende una serie de políticas e instituciones clave en la protección de estos segmentos de la población: el sistema de riesgos de trabajo (orientado a cubrir los accidentes y enfermedades laborales); el sistema previsional (a cargo de las jubilaciones y pensiones); el sistema de asignaciones familiares (que atiende la manutención de miembros del grupo familiar) y el seguro de desempleo. Como plantean Danani y Beccaria (2011, p.70), “a excepción de este último, creado en 1991, al comienzo del nuevo siglo todos los componentes de la Seguridad Social venían de atravesar una década de importantes transformaciones, que habían deteriorado su capacidad de protección social".

2 Las 24 obras sociales provinciales están agrupadas en el Consejo de Obras y Servicios Sociales Provinciales de la República Argentina (COSSPRA), institución civil sin fines de lucro que coordina acciones para garantizar cobertura de salud a 7.200 .000 afiliados. 
(De Ponti et al., 2016). Si bien en general son autárquicas suelen estar sujetas a las políticas de empleo, ingresos y a restricciones fiscales de los gobiernos subnacionales (Cetrángolo y Goldschmit, 2018, p. 15)

Por su volumen de asegurados la OSEP tiene un rol protagónico en el sistema de salud en Mendoza, una provincia con 1.800 .000 habitantes. Debe garantizar servicios a cerca de 400.000 personas (entre titulares y sus familias) que difieren en sus características socioeconómicas, posibilidades de acceso, expectativas y demandas de atención. En cuanto a su perfil demográfico, OSEP tiene una población con una marcada tendencia al envejecimiento $^{3}$, porque extiende sus protecciones a los estatales jubilados del estado provincial, situación que la diferencia de otras obras sociales provinciales y nacionales.

La provisión de servicios que presta es mixta: mediante convenios con privados y con el sector público y a través de infraestructura propia de la organización: tres hospitales, un centro odontológico, 18 sedes departamentales, unidades de atención de proximidad y programas preventivos ${ }^{4}$. La composición de los servicios, según la propiedad, varía significativamente en los territorios. Así, los asegurados que residen en zonas rurales o poco pobladas tienen como opciones de atención los servicios públicos de salud y los de propiedad de la organización mientras que quienes viven en zonas urbanas pueden acceder también a los servicios privados y de mayor complejidad que, en general, se concentran en base a criterios del mercado.

En este espacio complejo la OSEP promueve el desarrollo de investigaciones de las que participan diversos sectores y disciplinas presentes en la organización. La existencia de un comité de investigación y docencia, más de 40 residencias de posgrado, la publicación de una revista científica, el desarrollo de áreas que producen información institucional, son dispositivos con los que cuenta para generar conocimientos y formar profesionales receptivos a las complejidades del sector salud y a los desafíos políticos, financieros y epidemiológicos que enfrenta. No obstante, las iniciativas que desarrollan los diferentes equipos suelen autonomizarse de las prioridades organizacionales. A la vez, los decisores no siempre disponen de datos específicos para fijar prioridades sanitarias, “aun cuando son quienes están en las mejores condiciones para garantizar la integración de los resultados en tiempo real a fin de formular y poner en marcha políticas de salud y mejorar el desempeño de los sistemas de salud" (Langlois, Nhan, Ghaffar, Reveiz y Becerra-Posada, 2017, p. 1).

Si bien se reconoce que "las políticas de salud suelen estar más sujetas a los intereses de los artífices de políticas y a la presión que sobre ellos ejercen los contextos sociales,

3 Cerca del $13 \%$ de la población asegurada supera los 65 años. En departamentos urbanos del Gran Mendoza, la proporción de adultos mayores trepa al $20 \%$ del total.

4 En la organización trabajan 3.700 personas, de las cuales casi 2.000 son profesionales de la salud. 
políticos e intelectuales que a la influencia de las investigaciones" (Gómez, Orozco, Rodríguez y Velázquez, 2006, p. 114), se aspira a que las investigaciones perfeccionen sus decisiones. En especial atendiendo a que las organizaciones de salud “persiguen múltiples objetivos estratégicos de calidad en la práctica clínica, investigación, enseñanza, rentabilidad y eficiencia de los recursos y concordancia con la legislación vigente (LeónVanegas y González-Rodríguez, 2020, p. 5).

Atendiendo a lo expuesto, en noviembre de 2019 se propuso a referentes de distintos sectores de la OSEP, con diferente experiencia en gestión y con perfiles disciplinarios disímiles, participar de un proceso de construcción de consensos para identificar criterios y ejes de investigación a priorizar institucionalmente, en orden a "generar conocimiento para diseñar, implementar, analizar intervenciones y comprender el impacto de las mismas en la salud de la población" (Ministerio de salud y Desarrollo Social, 2019, p. 6).

Se optó por el método Delphi, en razón de su probada capacidad para generar acuerdos entre expertos sobre problemas del campo sanitario que se caracterizan por su complejidad, incertidumbre y por la falta de evidencias empíricas acerca de cómo resolverlos (García-Valdés y Suárez-Marín, 2013; Ministerio de salud y Desarrollo Social, 2019, p. 9; Varela-Ruiz, Díaz-Bravo y García-Durán, 2012; Yañez-Gallardo y Cuadra-Olmos, 2008).

Los objetivos de este escrito son difundir los resultados de la aplicación del método Delphi en la construcción consensuada de criterios y temas a investigar desde OSEP y ponderar las fortalezas de un enfoque cualitativo como el empleado, para producir conocimientos válidos para la planificación de los servicios de salud.

El artículo está estructurado de la siguiente manera: en el apartado metodológico se exponen las consideraciones epistemológicas, teóricas y operacionales que orientan el uso de la técnica elegida y las vicisitudes en su aplicación. Luego se presentan los resultados alcanzados, se discuten a la luz de otros trabajos y se cierra con conclusiones.

\section{Metodología}

La técnica Delphi integra el repertorio de las metodologías cualitativas. Como tal ofrece potencialidades para indagar procesos vinculados con la construcción de significados y valores de los equipos de trabajo y las disciplinas que convergen en los servicios de salud (Bowling, 2002; Mercado-Martínez, 2002; De Souza-Minayo, 2009) y para estudiar los conflictos y tensiones inherentes al diseño y la implementación de políticas sanitarias (Gilson, 2012; Pope, Ziebland \& Mays, 2000; Sheikh, 2012). 
En cuanto a su aplicación, la técnica Delphi supone convocar a discutir sobre un tópico controversial a expertos (conocedores de un tema), quienes deben emitir sus opiniones y/o respuestas en más de una ocasión, a través de varias rondas bajo la guía de un coordinador, hasta arribar a un consenso grupal. A partir de una secuencia iterativa, cada experto tiene la posibilidad de reconsiderar su opinión, a la luz de los planteamientos propios o de otros participantes, sin que ninguno sepa a quién corresponde tal o cual opinión (Varela-Ruiz et al., 2012; Yañez-Gallardo y Cuadra-Olmos, 2008). Con el anonimato se procura minimizar el predominio de unos participantes sobre otro/s, la influencia jerárquica en la emisión de juicios de valor o la inhibición de algunos de los convocados (Varela-Ruiz et al., 2012). Asimismo, la emisión de juicios en diferentes rondas a través de correos electrónicos facilita los esfuerzos de trabajo consensuado entre participantes de la red asistencial y entre funcionarios que tendrían limitaciones geográficas para reunirse o dificultades para acordar momentos en los que todos puedan trabajar juntos de manera presencial (Yañez-Gallardo y Cuadra-Olmos, 2008).

Teniendo en cuenta los requisitos y posibilidades de la técnica, se convocó a participar de un proceso de construcción de consensos en torno a las prioridades de investigación en OSEP a 26 referentes de diferentes áreas de la organización, quienes se desempeñan como directores en la administración central, en hospitales y a coordinadores de departamentos, programas y servicios territoriales con diferentes procedencias disciplinarias.

Tal como lo contempla la metodología Delphi, parar evita la aparición de sesgos en la información disponible en el panel, se procuró que los convocados "representaran una pluralidad en los planteamientos" (García-Valdés y Suárez-Marín, 2013) en torno a ¿para qué, cómo y qué investigar en OSEP?

La aplicación de la técnica se desarrolló en tres rondas a través del correo electrónico institucional entre el 1 y el 26 de noviembre de 2019; ningún miembro del grupo identificó a quién correspondió cada respuesta en particular en todo el proceso y en orden a preservar los anonimatos, las retroalimentaciones se enviaron por correo separado a cada panelista. Para sostener la participación de los convocados en las rondas sucesivas se desarrollaron estrategias de recordación a través de e-mail, WhatsApp y llamadas telefónicas.

Sobre los 26 expertos inicialmente convocados al panel, se consiguió el compromiso de colaboración efectivo de 20. Sostuvieron su participación y completaron las tres rondas 16 referentes. Aun con este desgranamiento se cumplió con la sugerencia señalada en la literatura según la cual "es aceptable la participación de entre 7 y 30 expertos" (ReguantÁlvarez y Torrado-Fonseca, 2016, p. 94). 
Las respuestas obtenidas tuvieron un tratamiento estadístico. Cuando se solicitaron estimaciones numéricas, se manejó la mediana de las respuestas individuales para conseguir la inclusión en el resultado final del grupo.

En la primera ronda se solicitó a los participantes que contestaran de manera sintética dos preguntas abiertas, a saber: 1) ¿Cuáles son las principales líneas de investigación que la Obra Social debería incentivar entre sus equipos de trabajo?, y 2) ¿Qué factores/criterios/requisitos se deberían contemplar para que la investigación opera como un insumo para mejorar la gestión de la organización?

Como resultado de la consulta inicial los participantes identificaron 72 líneas de investigación y 20 criterios y/o requisitos a considerar en los proyectos. Además, algunos enunciaron una serie de propuestas, que si bien no eran parte de la consigna original, se presentaron a los participantes y se exponen en este escrito.

Tras una primera sistematización de la información resultante en la primera vuelta, la coordinadora generó una segunda ronda de consultas, enviando a cada participante la totalidad de los juicios emitidos (sin identificar a sus emisores) con la nueva consigna de priorizar sólo 10 líneas de investigación y 10 requisitos y o criterios para desarrollar estudios en la OSEP. En esta segunda ronda, los participantes lograron un alto grado de consenso en torno a los atributos que deberían tener los proyectos de investigación realizados por equipos de la obra social que aspiren a lograr respaldo institucional. Las sugerencias para que las investigaciones operen como insumos para mejorar la gestión de la organización superaron en conjunto, el 50\% de acuerdo y las tres primeras tuvieron el aval de casi $90 \%$ de quienes respondieron.

A diferencia de los criterios de priorización, el tipo de problemas por investigar no alcanzó niveles mínimos de consenso en la segunda ronda de consultas. Hubo una altísima dispersión en las respuestas, lo que dio cuenta de una falta de criterios comunes básicos en torno a qué temas de investigación priorizar. Sobre 72 opciones planteadas en la primera ronda, 13 no fueron consideradas por nadie en la segunda (lo que refleja la apertura de sus propulsores frente a las propuestas del conjunto, que es lo que pretende tensionar esta metodología de trabajo), mientras que 41 problemas fueron priorizados por 1 o 2 participantes. Por lo cual, atendiendo al requisito de realimentación o feedback controlado, la coordinación transmitió los resultados y diseñó una nueva ronda de consultas. Esta vez se presentaron 19 problemas de investigación que superaron un mínimo de avales y se devolvieron ordenados en base a una ponderación que conjugó frecuencia de menciones y promedio del orden de prioridades asignadas por los participantes. Se solicitó que seleccionaran solo 5 problemas y los priorizaran calificándolos de 1 a 5 (sin repetir estos números), siendo 1 el más relevante y 5 el menos. Se transmitió además a través de mensajes por correo electrónico que se podían comentar los resultados de las dos consignas. Como 
producto en esta última ronda se manifestaron nuevas opiniones sobre los requisitos a tener en cuenta para investigar con el aval institucional de OSEP y se avanzó en la priorización de temas y ejes de investigación.

Los resultados obtenidos se discutieron con quienes completaron las tres rondas de consultas en un encuentro presencial en enero de 2020. En ese espacio se pusieron en valor los acuerdos alcanzados en materia de criterios a considerar en las investigaciones a promover desde OSEP. Se comentó que el proceso "posibilitó poner los intereses de la organización por encima de los propios" y "ayudar a reconsiderar lo que a cada uno puede resultarle interesante a la luz de intereses institucionales". Asimismo, los participantes rescataron la técnica desde la que se trabajó "porque ayuda a pensar", "resulta entretenida" y "permite intercambios" entre grupos de trabajo.

\section{Hallazgos}

Los criterios y requisitos acordados para priorizar proyectos de investigación en la organización fueron:

- Que los resultados de las investigaciones ofrezcan propuestas de solución viables a los problemas socio-sanitarios que enfrenta la obra social.

- Que el diseño de los proyectos (protocolos) respete los pasos formales, metodológicos y éticos de una buena investigación científica.

- Que los problemas a estudiar contribuyan a la sustentabilidad financiera de OSEP.

- Que favorezcan la transdiciplina y la interdisciplina.

- Que los resultados sean publicables y se contemple la devolución a la organización

- Que promuevan la eficiencia, eficacia y efectividad en OSEP.

- Que incentiven la transferencia del oficio de investigar de los profesionales con más experiencia a los que carecen de formación en investigación.

- Que contribuyan al posicionamiento institucional en el medio.

- Que sean factibles de realizar en los tiempos y con los recursos que provea OSEP.

- $\quad$ Que se funden en los valores: respeto, compromiso, diálogo y trabajo en equipo.

Salvo dos participantes, todos acordaron estos criterios y consensuaron el orden jerárquico en la segunda ronda. Un experto propuso invertir el orden sugiriendo que el primer lugar debe ser ocupado por el último criterio con el argumento de que "sin los valores mencionados en el punto $X$, no se pueden abordar el resto", y otro advirtió que: "difícilmente una investigación pueda reunir los diez criterios". Por lo que propuso "definir un punto de corte y, por ejemplo: si un proyecto reúne 8 de 10 criterios, esa investigación continúa en proceso de selección. O bien "definir criterios estrictos que si no están no aplica la investigación y criterios accesorios es decir que pueden no estar pero que suman cuando están presentes los criterios estrictos". 
Las líneas y problemas de investigación que institucionalmente OSEP debería priorizar, según lo consensuado por los participantes del Delphi fueron:

- Modelos de atención de la salud: prácticas innovadoras, atención centrada en personas

- Identificación de necesidades de afiliados que no acceden al sistema de servicios

- Acceso integral, oportuno y de calidad a servicios a partir del uso de tecnologías de la información, comunicaciones y biotecnología.

- Evaluación (en la línea de estudios de "evidencia del mundo real") de resultados de intervenciones de alto impacto económico que financia la obra social

- Calidad de la atención en atención primaria.

- Patologías de alta incidencia (y no necesariamente bajo costo)

- Prevención de riesgo cardio-metabólico

- Organización de respuestas asistenciales (cantidad, calidad, georreferenciación a servicios propios y externos) según necesidades de salud de grupos de afiliados

- Evaluación del impacto de la institucionalización (por falta de cuidados del entorno o falta de dispositivos alternativos) en la salud de los afiliados

- Caracterización de los hábitos y estilos de vida saludables de la población asegurada que contribuyan a la sustentabilidad financiera de OSEP en el mediano plazo

- Abordaje de la discapacidad: resultados de tratamientos, tratamientos alternativos, comparativa de calidad de vida antes y después de intervenciones; adecuación de los tratamientos a las necesidades de los niños con discapacidad e inclusión escolar

- Caracterización de las trayectorias profesionales de los egresados de las residencias de Osep y sus relaciones con las necesidades no cubiertas de recursos humanos en la organización.

Por último, las propuestas que validaron los participantes en el panel fueron:

- Realizar un curso de metodología de la investigación, nivelador para todos los trabajadores interesados.

- Definir un modelo de presentación de proyectos para unificar los estudios bajo la misma línea de investigación.

- Propiciar un registro de datos confiable

- Elaborar una agenda de conocimiento organizada por áreas o ejes estratégicos y que promuevan el trabajo interdisciplinario.

- Trabajar en una visión de futuro (prospectiva) donde se consoliden los lineamientos estratégicos propuestos en términos de investigación para el campo de interés.

- Crear en la web institucional un repositorio de investigaciones de la OSEP

- Presentar un portafolio de servicios de apoyo a la investigación dirigido a usuarios internos y externos (otras instituciones de salud o centros de investigación, 
empresarios, líderes de opinión, entidades relacionadas con el fomento de la investigación), que incluya acceso a bibliografía, a bases de datos y a textos completos de publicaciones académicas internacionales, acceso a diccionarios y a enciclopedias en línea.

- Apoyo a investigadores y a agentes para la presentación de ponencias orales, pósteres $\mathrm{u}$ otras modalidades de participación en eventos internacionales.

- Apoyar la traducción o corrección de estilo de artículos de investigación que vayan a ser sometidos a revistas con referato.

Los criterios consensuados acerca de los requisitos a contemplar en los estudios que se realicen con respaldo de la obra social a través del Delphi asumen que "el sentido de investigar en los servicios de salud es generar conocimiento, a través de la producción de nuevas ideas, para resolver problemas prácticos" (Manterola y Otzen, 2013 p. 1498). Se hacen eco también de la recomendación formulada por la Organización Panamericana de la Salud [OPS] y Organización Mundial de la Salud [OMS] (2009) cuando sostienen que:

La investigación emprendida para incrementar los conocimientos acerca de la salud. Incluye cualquier disciplina o combinación de disciplinas que procuren determinar y mejorar las repercusiones de las políticas, programas e intervenciones que se originan dentro y fuera del sector de la salud (...) y el estudio de sus relaciones con factores sociales, económicos, políticos (...), con el propósito de lograr el nivel más alto posible de salud. (p. 18)

Asimismo, los temas y problemas priorizados por los referentes de OSEP que participaron del panel tienen cierto correlato con temas y problemas difundidos por el Ministerio de Desarrollo Social y Salud de la Nación (MDSSN) en diciembre de 2019. Puntualmente cuando se explicitan las prioridades sanitarias por regiones, el documento nacional señala que las de la provincia de Mendoza deberían comprender:

...las respuestas de sistema de salud a las enfermedades crónicas no transmisibles (ECNT), barreras, equipos, redes de cuidados, derivación; la regionalización sanitaria y su relación con la salud materna, salud infantil y las ECNT; el fortalecimiento de la rehabilitación física, social y mental de personas con discapacidad y de las respuestas del sistema de salud ante emergencias, desastres y epidemias. (Ministerio de salud y Desarrollo Social, 2019 p. 4)

Ese mismo texto avanza sobre un listado de temas de alcance nacional y entre otros menciona la necesidad de incentivar investigaciones que indaguen:

Acceso, cobertura, capacidad prestacional y financiamiento de los sistemas de salud; modelos organizacionales y de gestión; Estrategia de APS; Enfermedades crónicas no transmisibles, Salud mental Rehabilitación y discapacidad, Salud Materno Infantil y 
Salud sexual y reproductiva; Atención integral al adulto mayor y Grupos vulnerables.

(Ministerio de salud y Desarrollo Social, 2019, pp. 57-58)

En contraste, entre los tópicos de investigación puestos en valor por el consenso nacional que no figuran entre las prioridades acordadas en el panel de OSEP figura el estudio de las enfermedades transmitidas por vectores, la atención integral a la familia y a la comunidad, los condicionantes y determinantes de la salud, violencia, inseguridad y accidentes, desigualdad, inequidad y pobreza.

Por último, las propuestas formuladas por los panelistas de OSEP están en sintonía con lo planteado en un documento internacional, fruto de un encuentro en El Cairo de 2008, que advertía que para que la investigación en salud sea eficiente y racional se necesita contar con metodologías válidas y confiables que permitan que los procedimientos para definir prioridades sean transparentes y objetivos (Ortiz et al., 2008).

Las propuestas emergentes del consenso en OSEP también se condicen con los lineamientos del Ministerio de Salud de Argentina que señala que: "las políticas de investigación para la salud deben promover la generación de investigaciones pertinentes, éticas y de calidad, la definición de temarios, mejorar la competencia de los recursos humanos que participan en la investigación y prestarles un mayor apoyo" (Ministerio de salud y Desarrollo Social, 2019, p. 6.).

Si bien todo lo expuesto clarifica la relevancia que la investigación tiene para la gestión sanitaria, no puede desconocerse que los directivos y los equipos de trabajo de las organizaciones de salud se enfrentan cotidianamente a presiones para decidir sobre cuestiones complejas que suelen implicar valores y metas en pugna.

El sector salud se desenvuelve en un entorno altamente dinámico, caracterizado por una creciente competencia y una necesaria contención de costos, debido a los cambios de las tendencias sociales en las últimas décadas, la demografía poblacional, las innovaciones tecnológicas, el aumento de la regulación estatal y los intereses en conflicto de los diferentes actores involucrados. Adicionalmente, deben ofrecer mejores resultados y una atención oportuna y de alta calidad. Lo anterior ha ocasionado que el cambio al que se enfrentan las organizaciones de cuidado de la salud sea más acelerado, en comparación con el que ocurre en muchos otros sectores. (León-Vanegas y González Rodríguez, 2020, p. 5)

Por otra parte, con relación a la aptitud de la técnica de investigación empleada para arribar a un consenso respecto a para qué, cómo y qué investigar en OSEP, constamos las ventajas que la literatura metodológica le atribuye al Delphi respecto de otras técnicas grupales. Es posible generar participación y acuerdos de manera no presencial y lograr que los juicios de los panelistas y el resultado final no sean obstruidos o sesgados por las 
posiciones y jerarquías individuales (Reguant-Álvarez y Torrado-Fonseca, 2016; VarelaRuiz et al., 2012). Esta cuestión no es menor cuando los participantes del estudio tienen diferente formación disciplinar (médicos, economistas, administradores, psicólogos, entre otros), diferentes niveles de responsabilidad en la organización e incluso diferente familiaridad con las decisiones y acciones que tienen lugar en materia de investigación.

Como se expuso en el apartado metodológico, si bien fue relativamente rápido acordar criterios comunes sobre los requisitos de la investigación, consensuar a qué problemas de investigación se les debería dar prioridad exigió a la coordinadora de la técnica una tercera ronda de consultas que no se había previsto. Esta situación contrasta con procesos iterativos descriptos en otros estudios Delphi donde la procedencia institucional de los expertos convocados y su relación temporal con el problema que se aborda es más disímil y sin embargo alcanzan acuerdos básicos en las primeras o segundas rondas. Por ejemplo otro estudio realizado en la provincia de Mendoza para indagar sobre la gestión hospitalaria en el contexto local, que convocó a altos mandos ministeriales y directores de hospitales que habían cumplido esa función entre 1983 y 2015, destacaba que, sobre más de 100 juicios emitidos en una primera ronda, los principales facilitadores y obstáculos para gobernar hospitales alcanzaron un consenso superior al $80 \%$ en la segunda ronda del estudio y fueron igualmente designadas por profesionales que habían estado involucrados con la dirección de hospitales de diferentes niveles de complejidad, con distintos marcos legales y bajo los lineamientos de gobiernos de diferente color político (Molina y Thomas, 2015, pp. 14-15). Es probable que el solapamiento de funciones de financiamiento, regulación y provisión, “los particulares mecanismos de compra y venta de servicios que se dirimen en los distintos niveles y que difieren según las prestaciones y las dinámicas territoriales" (Chiara, 2016, p. 19), así como la magnitud y heterogeneidad de la población a la que se atiende, condicione la disposición de los actores institucionales para pensarse como parte de un mismo todo. Si se acepta esta conjetura el proceso posibilitado por la técnica Delphi adquiere más valor, en tanto la realimentación permitió que "circulara información entre participantes y se avanzara hacia un lenguaje común en torno al sentido de investigar", que quizás hubiese sido difícil alcanzar apelando a otras técnicas (Varela-Ruiz et al., 2012). Al mismo tiempo que tensiona a los convocados a involucrarse gradualmente en un proceso de discriminación, selección y priorización de problemas importantes para todos los sectores (más allá de los propios) estimulando la generación de ideas compartidas en torno a un asunto controversial (García-Valdés y Suárez-Marín, 2013; Varela-Ruiz et al., 2012; Yañez-Gallardo y Cuadra-Olmos, 2008).

El proceso a la vez se adecúa a las asunciones ontológicas y epistemológicas de los enfoques cualitativos, en los cuales la validez de los estudios se construye como un ejercicio progresivo, de reflexión teórica y de diálogo y no como la búsqueda de correspondencia con una verdad única, como plantean las perspectivas positivistas (Cohen \& Crabtree, 2008, p. 337; Walt et al., 2008). El desafío asumido en la elección, coordinación y análisis de resultados 
de la metodología de consulta prospectiva y la forma de dar cuenta del proceso desarrollado reconoce una preocupación compartida entre quienes hacen investigación cualitativa en salud respecto de la necesidad de explicitar los detalles de los procedimientos, "la cocina", los retos metodológicos enfrentados ("la artesanía intelectual", según Mills) y la forma en que las posiciones del investigador pueden impactar sobre lo estudiado (Amezcua y GálvezToro, 2002; Marradi, 2002; Walt et al., 2008).

\section{Conclusiones}

Los resultados expuestos confirman que promover procesos sistemáticos de identificación de problemas que pueden ser resueltos mediante investigaciones contribuye a vislumbrar soluciones viables y sustentables a los desafíos asistenciales, financieros y de gestión que enfrentan las organizaciones de la seguridad social. La priorización de los problemas y las estrategias para estudiarlos se enriquecen en la medida en que se logra involucrar a los niveles directivos y a los equipos de trabajo en los procesos de análisis.

Las líneas de investigación que se reconocen como prioritarias responden a lecturas críticas sobre los límites que presentan ciertas modalidades de provisión de servicios asistenciales más centradas en las lógicas organizativas y disciplinares que en las características de sus destinatarios. Varios tópicos son puestos en valor a partir de la preocupación por sostener tratamientos integrales, sobre todo frente a problemáticas complejas como la discapacidad o las enfermedades crónicas. En este sentido, aparece como un imperativo promover investigaciones que permitan evaluar los resultados de los dispositivos de atención en términos de garantías de acceso y de continuidad asistencial a segmentos de poblaciones que, aun cuando compartan diagnósticos o patologías determinadas, son heterogéneas en sus condiciones de vida y en sus posibilidades de vincularse con el sistema de servicios.

Por otra parte, los consensos expuestos dan cuenta de que la sustentabilidad financiera de las aseguradoras de salud resulta clave para atender a poblaciones en proceso de envejecimiento y con altas tasas de cronicidad. Se advierte en los debates que la preocupación por la sustentabilidad se vincula con innovaciones en las lógicas de cuidado que incorporen, entre otras cuestiones, un uso más intensivo de nuevas tecnologías de atención, como la teleasistencia. Al mismo tiempo, se pone el foco en los modos de vida y en las dimensiones territoriales que condicionan la salud y que se dirimen dentro y fuera de los servicios médicos. En esta línea, parece necesario motivar a los directivos y a los equipos de salud para que estudien qué adecuaciones podrían incorporarse a los esquemas de cuidados para mayor receptividad y adherencia a los tratamientos $\mathrm{y}$, de ese modo, avanzar en eficacia e inclusión social. 
El apoyo del método Delphi como herramienta para identificar y consensuar criterios y problemas a investigar en los servicios de salud facilita que actores que no necesariamente trabajan de manera articulada reconsideren intereses particulares a la luz de intereses institucionales. Al mismo tiempo, aporta información a las áreas de investigación y docencia y a los gestores sobre la base de un procedimiento reflexivo, riguroso y fundado.

Sin desconocer que las decisiones en materia de política sanitaria están atravesadas por valores contradictorios y por múltiples intereses y constricciones que complejizan el logro de mejoras en la calidad de vida de las poblaciones, se considera que procesos participativos de priorización de líneas investigativas como el desarrollado promueven miradas interdisciplinarias para revisar el presente y el futuro de las organizaciones de salud. Para que los hallazgos que resultan de la construcción de consensos permeen la dinámica cotidiana de los servicios y los niveles de decisión es recomendable convocar al intercambio de ideas a profesionales y gestores con diversos perfiles, trayectorias y posiciones en el espacio institucional y, a la vez, comprometidos en promover la generación de nuevos conocimientos para alcanzar mejoras en el acceso a la salud.

\section{Referencias bibliográficas}

Amezcua, M., y Gálvez-Toro, A. (2002). Los modos de análisis en investigación cualitativa en salud: perspectiva crítica y reflexiones en voz alta. Revista Española de Salud Pública, 76(5), 423-436. Recuperado de http:/ / scielo.isciii.es/pdf/resp/v76n5/colabora4.pdf.

Bowling, A. (2002). Research methods in health. Investigating health and health services. (2 ed.). Buckingham: Open University Press.

Cetrángolo, O., y Goldschmit, A. (2018). Las obras sociales y otras instituciones de la Seguridad social para la salud en Argentina. Origen y situación actual de un sistema altamente desigual. Buenos Aires, Argentina: Fundación CECE. Recuperado de http:/ / fcece.org.ar/wp-content/uploads/informes/obras-sociales-argentina.pdf.

Chiara, M. (2016). Territorio, políticas públicas y salud. Hacia la construcción de un enfoque multidimensional para la investigación. Revista Gerencia y Políticas de Salud, 15(30), 10-22. Recuperado de 10.11144/Javeriana.rgyps15-30.tpps.

Cohen, D., \& Crabtree, B. (2008). Evaluative criteria for qualitative research in health care: controversies and recommendations. The Annals of Family Medicine, 6(4), 331-339. doi: $10.1370 / \mathrm{afm} .818$.

Danani, C., y Beccaria, A. (2011). Seguridad Social: marchas y contramarchas en la protección, la seguridad y el reconocimiento de derechos, 2002-2011. Voces en el Fenix, (6), 68-73.

García-Valdés, M., y Suárez-Marín, M. (2013). El método Delphi para la consulta a expertos en la investigación científica. Revista Cubana de Salud Pública, 39(2), 253-267. 
Recuperado de https://www.medigraphic.com/pdfs/revcubsalpub/csp2013/csp132g.pdf.

Gilson, L. (Ed.). (2012). Health policy and systems research: A methodology reader. Geneve: World Health Organization. Alliance for Health Policy and Systems Research. Recuperado de https://www.who.int/alliance-hpsr/resources/reader/en/.

Gómez, R. D., Orozco, D., Rodríguez, F., y Velázquez, W. (2006). Políticas públicas y salud: relación entre investigación y decisión. Revista Facultad Nacional de Salud Pública 24(2), 105-118. Recuperado de http://www.scielo.org.co/pdf/rfnsp/v24n2/v24n2a11.pdf.

Langlois, E. V., Nhan, T. T., Ghaffar, A., Reveiz, L., \& Becerra-Posada, F. (2017). Embedding research in health policy and systems in the Americas. Revista Panamericana de Salud Pública, 41, e68. Recuperado de https:/ / iris.paho.org/bitstream/handle/10665.2/34037/v41a682017.pdf?sequence= 8\&isAllowed $=\mathrm{y}$.

León-Vanegas, Y. L., y González-Rodríguez, J. L. (2020). La toma de decisiones estratégicas en organizaciones de salud. Una revisión de la literatura. Revista Gerencia y Políticas de Salud, 19, 1-18. doi: 10.11144/Javeriana.rgps19.tdeo.

Manterola, C., y Otzen, T. (2013). Porqué investigar y cómo conducir una investigación. International Journal of Morphology, 31(4), 1498-1504. doi: doi: 10.4067/S071795022013000400056.

Marradi, A. (2002). Método como arte. Papers Revista de sociología, 67, 107-127 Recuperado de https:/ / papers.uab.cat/article/view/v67-marradi/pdf-es.

Mercado-Martínez, F. J. (2002). Investigación cualitativa en América Latina: perspectivas críticas en salud. International Journal of Qualitative Methods, 1(1), Article 4. Recuperado

de https:/ / sites.ualberta.ca/ iiqm/backissues/1_1Final/pdf/mercadospanish.pdf.

Ministerio de salud y Desarrollo Social. (2019). Agenda Nacional de Investigación en Salud Pública Establecimiento de Prioridades. Buenos Aires: Ministerio de salud y Desarrollo Social. Secretaria de Gobierno de Salud. Dirección de Investigación para la salud. Recuperado de http://iah.salud.gob.ar/doc/435.pdf.

Molina, C., y Thomas, C. (2015). ¿Por qué es difícil gobernar hospitales públicos? La mirada de los gestores en Mendoza. Mendoza: Instituto de Salud Pública y Gestión sanitaria.

Organización Panamericana de la Salud [OPS] y Organización Mundial de la Salud [OMS]. (2009). Política de Investigación para la Salud. Recuperado de https://www.paho.org/hq/dmdocuments/2009/CD49-10-s.pdf.

Ortiz, Z., Olmos, M., Ramos, S., Sosa-Estani, S., Kochenm, S., Pecheny, M. ... Segura, E. L. (January, 2008). Setting health research priorities in Argentina: the combined Approach Matrix. In Forum 10: Annual meeting. Global Forum for Health Research, Cairo, Egypt.

Ponti, H. de, Canale, S., y Monteferrario, M. (setiembre, 2016). Fragmentación de cobertura de servicios de salud. El caso de las Obras Sociales Provinciales. En Jornadas de 
economía de la salud 2016: “Crisis económica, inversión y sustentabilidad del sector salud en Latinoamérica y Caribe". Universidad ISALUD, Ciudad Autónoma de Buenos Aires, Argentina. Recuperado de http:/ / www.oossp.com.ar/archivos/pubs/fragmentac\%20cob\%20y\%20osp \%20aes \%202016.pdf.

Pope, C., Ziebland, S., \& Mays, N. (2000). Qualitative research in health care. Analysing qualitative data. British Medical Journal, 320(7227), 114-116. doi: 10.1136/bmj.320.7227.114.

Reguant-Álvarez, M., y Torrado-Fonseca, M. (2016). El método Delphi. REIRE, Revista d'Innovació i Recerca en Educació, 9(1), 87-102. doi: 10.1344/reire2016.9.1916.

Sheikh, K. (2012). Analyzing power in health systems: the case of medical dominance in India. BMC Proceedings, 6(Suppl 5), O6. doi: 10.1186/1753-6561-6-S5-O6.

Souza-Minayo, M. C. de. (2009). La artesanía de la investigación cualitativa. Buenos Aires, Argentina: Lugar Editorial.

Varela-Ruiz, M., Díaz-Bravo, L., y García-Durán, R. (2012). Descripción y usos del método Delphi en investigaciones del área de la salud. Investigación en Educación Médica, 1(2), 90-95. Recuperado de https://www.elsevier.es/es-revista-investigacioneducacion-medica-343-pdf-X2007505712427047.

Walt, G., Shiffman, J., Schneider, H., Murray, S., Brugha, R., \& Gilson, L. (2008). 'Doing' health policy analysis: methodological and conceptual reflections and challenges. Health Policy and Planning, 23(5), 308-317. doi: 10.1093/heapol/czn024.

Yañez-Gallardo, R., y Cuadra-Olmos, R. (2008). La técnica Delphi y la investigación en los servicios de salud. Ciencia y enfermería, 14(1), 9-15. doi: 10.4067/S071795532008000100002. 


\section{OTROS ARTÍCULOS DE PROSPECTIVA No. 32 DE 2021}

\section{EDITORIAL}

Compromisos, dilemas y desafíos del Trabajo Social con dimensión colectiva en tiempos de pandemia

Enrique Pastor-Seller

\section{ARTÍCULOS}

La narrativa biográfica como alternativa para la comprensión del Conflicto y la violencia en Colombia: una experiencia pedagógica universitaria

Sara Sofía Castaño-Barco y Claudia BermúdezPeña

Experiencias de transición a la vida adulta de jóvenes que migraron solos. Un estudio en Aragón (España) Daniel Jiménez-Franco; Raquel Berzosa-Callén y Chabier Gimeno-Monterde

Fundamentals of Education in Intervention with Young Offenders. A Critical Review of a 'Successful' Case in Andalucía, España

David Herrera-Pastor

Subjetividad juvenil: lecturas desde y para el Trabajo Social en Colombia

Daniela Joya-Valbuena

Participación politica de los jóvenes del Valle del Cauca, Colombia, en la toma de decisiones públicas

Luis Eduardo Gutiérrez-Rojas y Amanda Ramírez-Giraldo

Victimización y perpetración de violencia en pareja adolescente y redes de apoyo en Colombia. Análisis con perspectiva de género

Johanna Alexandra Reina-Barreto
Apoyo social percibido, autoestima y maternidad adolescente: entre el respeto y la intrusión. Estudio en Traiguén, Chile

Gloria Mora-Guerrero, Luisa Escárate-Colín, Carol Espinoza-Lerdón y Andrea Peña-Paredes

El desarrollo en el Trabajo Social de Colombia: un campo transversal, disperso y polivalente

Maira Judith Contreras-Santos

Análisis de la situación laboral de las alimentadoras en la caficultura de la zona central colombiana, a la luz de la teoría de Nancy Fraser

Pablo Andrés Arango-Giraldo

Prioridades de investigación en la Obra social de empleados públicos, Mendoza, Argentina. Un estudio Delphi sobre producción de conocimientos en la seguridad social

Cecilia Amalia Molina

Producción de conocimiento y toma de decisiones. Relaciones entre academia y política pública para las familias en Colombia

José Raúl-Ruíz y Sandro Leonardo MunévarVargas

El staff de prácticas profesionales en Trabajo Social: espacio de fomento del aprendizaje autodirigido. Un estudio de caso en la Universidad Católica Luis Amigó, Colombia

Cristian Eduardo Blanco-García

\section{ARTISTA INVITADO}

Margie Reinel-Aguilar Ao Cicadidae

Prospectiva

\section{PROSPECTIVA Revista de Trabajo Social e Intervención Social}

No. 32 • jul.-dic. 2021

e-ISSN: 2389-993X • Universidad del Valle 